\title{
Interpreting Stochastic Frontier Model as Random Coefficient Model and Vice Versa
}

\author{
Spyros Missiakoulis ${ }^{1}$
}

\begin{abstract}
This note explores the relationship between the stochastic frontier model and the random coefficient regression model. It shows how to interpret the former as a special case of the latter and vice versa.
\end{abstract}

JEL classification numbers: C13, C51, D24.

Keywords: Stochastic production frontier, Random coefficient regression, Composite error, Technical inefficiency.

${ }^{1}$ Philips University, Nicosia, Cyprus. 


\section{The Stochastic Frontier Production Model}

The stochastic frontier production model introduced by Aigner, Lovell and Schmidt (1977) and independently by Meeusen and Broeck (1977) relates the production function

$$
y_{i}=\beta_{0}+\sum_{j=1}^{k} \beta_{j} x_{i j}+\varepsilon_{i}
$$

with the composite error specification

$$
\varepsilon_{i}=v_{i}-u_{i}
$$

where $y_{i}$ measures the output, $x_{i j}$ measures the $j^{\text {th }}$ input, $\beta_{0}$ is the unknown intercept, $\beta_{j}$ is the $j^{\text {th }}$ unknown slope parameter, $v_{i}$ represents the usual two-sided distribution term with $v_{i} \sim N I D\left(0, \sigma_{v}^{2}\right)$, and $u_{i}$, which is distributed independently of $v_{i}$ and satisfies $u_{i} \geq 0$, represents the technical errors of the firm. The most commonly assumed distribution of $u_{i}$ is the (positive) half-normal. This is not a restrictive assumption as any other suitable (positive) distribution of $u_{i}$ may be considered. The technical inefficiency relative to the stochastic frontier is then given by $u_{i} \%$. Model (1) with (2) will be referred to as the ALS model. Excellent reviews of the ALS model and the frontier literature, in general, may be found in Forsund et al. (1980) and, Kumbhakar and Lovell (2000).

All unknown parameters of the ALS model are estimated either by Corrected Ordinary Least Squares, or Maximum Likelihood (Aigner et al. 1977; Olson et al. 1980; Lee 1983; Greene 1990; Greene 2010), with each method having its pros and cons.

The main weakness of the ALS model was the impossibility to determine the individual technical inefficiencies. Although the mean technical inefficiency over the sample is estimable, it was not possible to decompose each residual into its two components. Jondrow et al. (1982) suggested a solution to this problem by considering the expected value of $u_{i}$, conditional upon $\varepsilon_{i}$. Under the half-normal assumption for $u_{i}$, individual coefficients are estimated either by

$$
u_{i}=\frac{\sigma_{u} \sigma_{v}}{\sqrt{\sigma_{u}^{2}+\sigma_{v}^{2}}}\left(\frac{f\left(z_{i}\right)}{1-F\left(z_{i}\right)}\right)
$$

where

$$
z_{i}=\frac{\varepsilon_{i} \sigma_{u}}{\sigma_{v} \sqrt{\sigma_{u}^{2}+\sigma_{v}^{2}}}
$$

and $f(\cdot)$ and $F(\cdot)$ are the standard normal density and distribution functions respectively; or by

$$
\begin{aligned}
& u_{i}=\frac{\sigma_{u}^{2}}{\sigma_{u}^{2}+\sigma_{v}^{2}} \varepsilon_{i} \quad \text { if } \quad \varepsilon_{i} \leq 0 \\
& =0 \quad \text { if } \varepsilon_{i}>0 \text {. }
\end{aligned}
$$

The former is based on the mean of $u_{i}$ conditional on $\varepsilon_{i}$, and the latter is based on the mode of $u_{i}$ conditional on $\varepsilon_{i}$. 


\section{The Random Coefficient Model}

The conventional random coefficient regression model is given by

with

$$
y_{i}=\beta_{i 0}+\sum_{j=1}^{k} \beta_{i j} x_{i j}
$$

$$
\beta_{i j}=\beta_{j}+w_{i j} \quad j=0,1, \ldots, k
$$

where all the $w$ 's are assumed as $w_{i j} \sim \operatorname{NID}\left(0, \sigma_{j}^{2}\right), j=0, \ldots, k$. Note, if it is desired, an error term may be also introduced in (5). We may now rewrite (5) as

$$
y_{i}=\beta_{0}+\sum_{j=1}^{k} \beta_{j} x_{i j}+\varepsilon_{i}
$$

where now

$$
\varepsilon_{i}=w_{i 0}+\sum_{j=1}^{k} x_{i j} w_{i j}
$$

Maximum Likelihood and Generalized Least Squares methods have been suggested for the estimation of $\beta_{j}, j=0, \ldots, k$ and their corresponding variances (Swamy, 1971; Raj and Ullah, 1981; Kreft and de Leeuw 1998).

\section{The equivalence of the two models}

Consider now the random coefficient model in (5) or (7) with only one difference, namely, $w_{i 0}$ in (6) or (8) is not normally distributed but follows the one-sided distribution of $-u_{i}$ in (2). In other words, by setting $w_{i 0}=-u_{i}$ and $\Sigma x_{i j} w_{i j}=v_{i}$ the model in (7) and (8) is nothing else but the ALS model. Of course, we may argue the other way. That is if we assume that $u_{i}=-w_{i 0}$ and $v_{i}=$ $\Sigma x_{i j} w_{i j}$, the ALS model is a special case of the random coefficient regression model. Since each $w_{i j}, j=1, \ldots, k$, is normally distributed, then $v_{i}$ is also normally distributed.

Griffiths (1972) showed that under the assumption, each residual is allocated between its components in the same proportion as the variance of $\varepsilon_{i}$ is allocated between the variance of each error component, GLS estimates of each individual coefficient are minimum variance, linear, unbiased estimates.

Adopting Griffiths' assumption we may construct technical inefficiencies as

$$
\begin{aligned}
& u_{i}=-\frac{\operatorname{Var}\left(u_{i}\right)}{\operatorname{Var}\left(u_{i}\right)+\operatorname{Var}\left(v_{i}\right)} \varepsilon_{i}=-\frac{(\pi-2) \sigma_{u}^{2}}{(\pi-2) \sigma_{u}^{2}+\pi \sigma_{v}^{2}} \varepsilon_{i} \quad \text { if } \quad \varepsilon_{i} \leq 0 \\
& =0 \quad \text { if } \varepsilon_{i}>0 \text {, }
\end{aligned}
$$

If, however, we also adopt the ALS interpretation that the relative variability of the two sources of random error is a function of $\sigma_{u}^{2}$ and $\sigma_{v}^{2}$ and not of $\operatorname{Var}\left(u_{i}\right)$ and $\operatorname{Var}\left(v_{i}\right)$ then (9) is written as

$$
\begin{aligned}
& u_{i}=\frac{\sigma_{u}^{2}}{\sigma_{u}^{2}+\sigma_{v}^{2}} \varepsilon_{i} \quad \text { if } \quad \varepsilon_{i} \leq 0 \\
& =0 \quad \text { if } \varepsilon_{i}>0 \text {, }
\end{aligned}
$$

which is exactly the same as (4).

Although this note focuses on the frontier production model, its generalization for all frontier models, i.e. cost or profit frontiers, is trivial. 


\section{References}

[1] Aigner, D.J., Lovell, C.A.K. and Schmidt, P. (1977). Formulation and Estimation of Stochastic Frontier Production Models. Journal of Econometrics, 6, pp. 21-37.

[2] Forsund, F.R., Lovell, C.A.K. and Schmidt, P. (1980). A survey of frontier production functions and their relationship to efficiency measurements. Journal of Econometrics, 13, pp. 5-26.

[3] Greene, W.H. (1990). A gamma distributed stochastic frontier model. Journal of Econometrics, 46, pp. 141-164.

[4] Greene, W.H. (2010). A stochastic frontier model with correction for sample selection. Journal of productivity analysis, 34, pp. 15-24.

[5] Griffiths, W.E. (1972). Estimation of actual response coefficients in the Hildreth-Houck random coefficient model. Journal of the American Statistical Association, 67, pp. 633635.

[6] Jondrow, J., Lovell, C.A.K., Materov, I.S. and Schmidt, P. (1982). On the estimation of technical inefficiency in the stochastic frontier production function model. Journal of Econometrics, 19, pp. 233-238.

[7] Kreft, I.G. and de Leeuw, J. (1998). Varying and random coefficient models. In Introducing multilevel modeling, SAGE Publications, pp. 35-56.

[8] Kumbhakar, S. and Lovell, C.A.K. (2000). Stochastic Frontier Analysis. Cambridge University Press, Cambridge.

[9] Lee, L.F. (1983). On maximum likelihood estimation of stochastic frontier production models. Journal of Econometrics, 23, pp. 269-274.

[10] Meeusen, W. and van den Broeck, J. (1977). Efficiency estimation from Cobb-Douglas production functions with composed errors. International Economic Review, 18, pp. 435444.

[11] Olson, J.A., Schmidt, P. and Waldman M. (1980). A Monte Carlo study of estimators of stochastic frontier production functions. Journal of Econometrics, 13, pp. 67-82.

[12] Raj, B. and Ullah, A. (1981). Econometrics: A Varying Coefficient Approach, Croom Helm, London.

[13] Swamy, P.A.V.B. (1971). Statistical Inference in Random Coefficient Regression Models, Springer-Verlag, Berlin. 